\title{
Discrete circles: an arithmetical approach with non-constant thickness
}

\author{
C. Fiorio, D. Jamet and J.-L. Toutant \\ LIRMM-UMR 5506, Université Montpellier II, \\ 161 rue Ada, \\ 34392 Montpellier Cedex 5 - FRANCE.
}

\begin{abstract}
In the present paper, we introduce an arithmetical definition of discrete circles with a non-constant thickness and we exhibit different classes of them depending on the arithmetical discrete lines. On the one hand, it results in the characterization of regular discrete circles with integer parameters as well as J. Bresenham's circles. As far as we know, it is the first arithmetical definition of the latter one. On the other hand, we introduce new discrete circles, actually the thinnest ones for the usual discrete connectedness relations.
\end{abstract}

Keywords: arithmetical discrete geometry, discrete circle, discrete curve, Bresenham, norms

\section{INTRODUCTION}

Discrete geometry attempts to provide an analogue of euclidean geometry for the discrete space $\mathbb{Z}^{n}$. Such an investigation is not only theoretical, but has also practical applications since digital images can be seen as arrays of pixels, in other words, subsets of $\mathbb{Z}^{2}$.

Since the seventies, the discrete lines, namely the analogue of euclidean lines in the discrete space $\mathbb{Z}^{n}$, have been widely studied. On the one hand, J. Bresenham, ${ }^{3}$ A. Rosenfeld ${ }^{15}$ and H. Freeman ${ }^{7}$ followed an algorithmical approach and defined discrete lines as digitizations of euclidean ones. They provide tools for drawing and recognition. ${ }^{7,8,15}$ On the other hand, J.-P. Reveillès initiated the discrete arithmetical geometry ${ }^{14}$ and introduced the arithmetical discrete lines as subsets of $\mathbb{Z}^{2}$ satisfying a double diophantine inequality (see Definition 3). Such an approach enhances the knowledge of the discrete lines. In addition to give new drawing ${ }^{14}$ and recognition ${ }^{5}$ algorithms, it directly links topological and geometrical properties of an arithmetical discrete line with its definition. For instance, the connectedness of an arithmetical discrete line is entirely characterized (see Theorem 1).

The other well-studied family of discrete objects is the one of discrete circles. Similarly as in the case of discrete lines, the first investigations were algorithmical. ${ }^{4,10,11,13}$ Discrete circles were considered as digitizations of euclidean ones. It is thus natural to ask whether J.-P. Reveillès' arithmetical approach is extendable to discrete circles, in order to supply them with a definition independent of euclidean geometry. If so, one can expect discrete circles to share topological and geometrical properties with euclidean circles:

(i) the connectedness: a discrete circle is $k$-connected (see Definition 1 ),

(ii) the thinness: a discrete circle is $(1-k)$-minimal (see Definition 2 ),

(iii) the tiling of the space: concentric discrete circles tile the discrete space $\mathbb{Z}^{2}$.

Further author information: (Send correspondence to J.-L.T.)

C.F.: E-mail: fiorio@lirmm.fr,

D.J.: E-mail: jamet@lirmm.fr,

J.-L.T.: E-mail: toutant@lirmm.fr. 
Such an extension has been proposed by É. Andres. ${ }^{1}$ His discrete analytical circles, defined as subsets of $\mathbb{Z}^{2}$, verify a double diophantine inequality (see Definition 5 ) and are provided with a constant thickness, similarly to J.-P. Reveillès' arithmetical discrete lines. ${ }^{14}$ In other words, a discrete analytical circle is nothing but a discrete ring, that is, a set a pixels at a bounded distance from an euclidean circle. In that case, one shows that the condition (iii) holds while (i) and (ii) are partially satisfied. ${ }^{1}$

In the present paper, in order to deal with the properties of circles, we introduce an arithmetical definition of discrete circles with a non-constant thickness. In particular, we investigate different approximations of circles by discrete lines. Depending on the nature (naive, standard, ...) of these lines, we characterize new discrete circles, such as the $k$-minimal ones (see Definition 2), or already known discrete circles, such as the regular ones with integer parameters ${ }^{1}$ or the Bresenham's ones. ${ }^{4}$ As far as we know, this is the first arithmetical characterization of the latter.

This paper is sketched as follows:

- We begin with some recalls. First, Section 2 gives basic notions on discrete connectedness useful to fully understand the present paper. Second, The arithmetical discrete lines which inspire our approach are presented in Section 3. Finally, in Section 4, we focus on already known discrete circles.

- In Section 5, we introduce a general arithmetical definition of discrete curves extending the artihmetical discrete lines.

- In Section 6, we apply our general definition to the particular case of discrete circles. we characterize the already known circles of Section 4 and define new classes fo discrete circles.

\section{BASIC NOTIONS}

The aim of this section is to introduce the basic notions of discrete geometry which are used all along the present paper. Let $n$ be an integer greater than 2 and let $\left\{\mathbf{e}_{\mathbf{1}}, \ldots, \mathbf{e}_{\mathbf{n}}\right\}$ denote the canonical basis of the euclidean vector space $\mathbb{R}^{n}$. Let us call discrete set any subset of the discrete space $\mathbb{Z}^{n}$. If the point $\mathbf{M}=\sum_{i=1}^{n} M_{i} \mathbf{e}_{\mathbf{i}} \in \mathbb{R}^{n}$, with $M_{i} \in \mathbb{R}$ for each $i \in\{1, \ldots, n\}$, then $\mathbf{M}$ is represented by $\left(M_{1}, \ldots, M_{n}\right)$. A point of $\mathbb{Z}^{n}$ is called a voxel. If $n=2$, a voxel is also called a pixel.

Let $\mathbf{N}=\left(N_{1}, \ldots, N_{n}\right)$ and $\mathbf{N}^{\prime}=\left(N_{1}^{\prime}, \ldots, N_{n}^{\prime}\right)$ be two voxels and let $k \in\{0, \ldots, n-1\}$. The voxels $\mathbf{N}$ and $\mathbf{N}^{\prime}$ are said to be $k$-neigbors if and only if:

$$
\left\|\mathbf{N}-\mathbf{N}^{\prime}\right\|_{\infty}=\max \left\{\left|N_{1}-N_{1}^{\prime}\right|, \ldots,\left|N_{n}-N_{n}^{\prime}\right|\right\}=1 \text { and }\left\|\mathbf{N}-\mathbf{N}^{\prime}\right\|_{1}=\sum_{i=1}^{n}\left|N_{i}-N_{i}^{\prime}\right| \leq n-k .
$$

Definition 1 ( $k$-connectedness) Let $k \in\{0, \ldots, n-1\}$. A discrete set $\mathbb{E}$ is said to be $k$-connected if for each pair $\left(\mathbf{N}, \mathbf{N}^{\prime}\right)$ of voxels in $\mathbb{E}$, there exists a finite sequence $\left(\mathbf{N}_{1}, \ldots, \mathbf{N}_{\mathbf{p}}\right)$ of voxels such that $\mathbf{N}=\mathbf{N}_{\mathbf{1}}, \mathbf{N}^{\prime}=\mathbf{N}_{\mathbf{p}}$ and the voxels $\mathbf{N}_{\mathbf{j}}$ and $\mathbf{N}_{\mathbf{j}+\mathbf{1}}$ are $k$-neighbors, for each $j \in\{1, \ldots, p-1\}$. For clarity issues, a 0 -connected discrete set is said to be connected.

Let $\mathbb{E}$ be a discrete set, $\mathbf{N}$ be a voxel of $\mathbb{E}$ and $k \in\{0, \ldots, n-1\}$. The $k$-connected component of $\mathbf{N}$ in $\mathbb{E}$ is the maximal $k$-connected subset of $\mathbb{E}$, for inclusion, containing $\mathbf{N}$. The discrete set $\mathbb{E}$ is said to be $k$-separable if its complement $\overline{\mathbb{E}}=\mathbb{Z}^{n} \backslash \mathbb{E}$ has two $k$-connected components.

Definition $2(k$-simple points, $k$-minimality) Let $k \in\{0, \ldots, n-1\}$. A voxel $\mathbf{N}$ of a discrete set $\mathbb{E}$ is said to be $k$-simple in $\mathbb{E}$ if $\mathbb{E} \backslash\{\mathbf{N}\}$ is $k$-separable. A $k$-separable discrete set without $k$-simple points is said to be k-minimal.

In the present paper, we work in the two-dimensional discrete space $\mathbb{Z}^{2}$ for clarity issues but all the ideas and definitions presented extend in a natural way to $\mathbb{Z}^{n}$. 


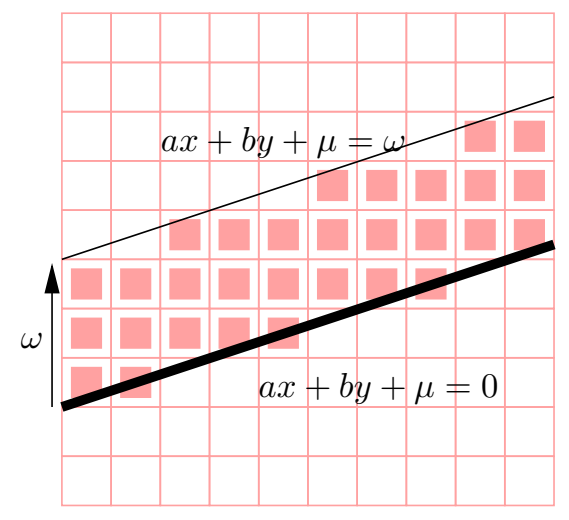

(a) General definition.

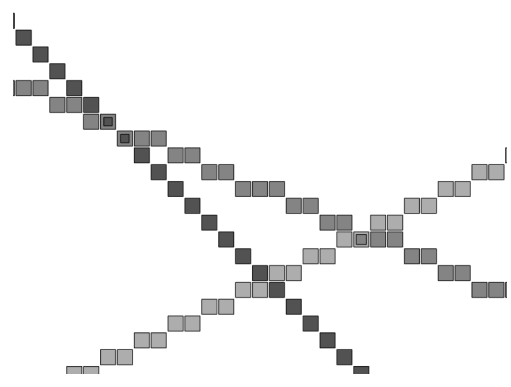

(b) Naive discrete lines.

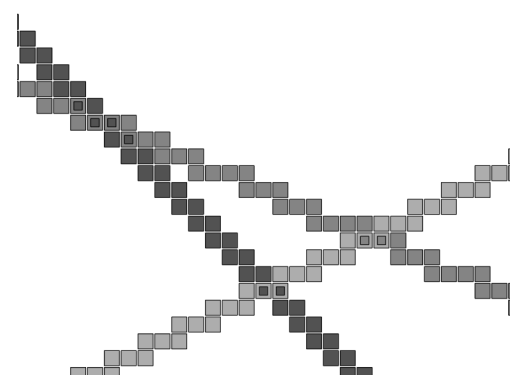

(c) Standard discrete lines.

Figure 1. Arithmetical discrete lines.

\section{ARITHMETICAL DISCRETE LINES}

In the early nineties, J.-P. Reveillès ${ }^{14}$ has laid the foundations of a new geometry on $\mathbb{Z}^{n}$, the arithmetical discrete geometry. Its link with the usual euclidean geometry takes place at infinity*: a discrete lattice seen from an infinitely distant point seems to be continuous. Unfortunately, due to the discrete character of $\mathbb{Z}^{n}$, a single continuous object has several discrete representations, each one inheriting some of its properties. For instance, arithmetical discrete lines are defined as strips depending on a parameter $w \in \mathbb{N}$, the so-called arithmetical thickness (see Figure 1(a)).

Definition 3 (Arithmetical discrete lines ${ }^{14}$ ) The arithmetical discrete line $\mathfrak{D}(\mathbf{v}, \mu, \omega)$ of normal vector $\mathbf{v}=$ $(a, b) \in \mathbb{R}^{2}$, translation parameter $\mu \in \mathbb{R}$ and arithmetical thickness $\omega \in \mathbb{R}_{+}$is the subset of $\mathbb{Z}^{2}$ defined by:

$$
\mathfrak{D}(\mathbf{v}, \mu, \omega)=\left\{(i, j) \in \mathbb{Z}^{2} \mid-\frac{\omega}{2} \leq a i+b j+\mu<\frac{\omega}{2}\right\}
$$

The choice of the inequalities in (1) is arbitrary, one thus can choose to define the arithmetical discrete line $\widetilde{\mathfrak{D}}(\mathbf{v}, \mu, w)$ of vector normal $\mathbf{v}=(a, b) \in \mathbb{R}^{2}$, translation parameter $\mu \in \mathbb{R}$ and arithmetical thickness $\omega \in \mathbb{R}_{+}$as follows:

$$
\widetilde{\mathfrak{D}}(\mathbf{v}, \mu, \omega)=\left\{(i, j) \in \mathbb{Z}^{2} \mid-\frac{\omega}{2}<a i+b j+\mu \leq \frac{\omega}{2}\right\} .
$$

The arithmetical discrete line $\mathfrak{D}(\mathbf{v}, \mu, \omega)$ (resp. $\widetilde{\mathfrak{D}}(\mathbf{v}, \mu, \omega))$ is called the lower (resp. the upper) arithmetical discrete line of normal vector $\mathbf{v} \in \mathbb{R}^{2}$, translation parameter $\mu \in \mathbb{R}$ and arithmetical thickness $\omega \in \mathbb{R}_{+}$

In fact, an arithmetical discrete line is the set of solutions of diophantine equations, that is, the ones with integer solutions. Given an arithmetical discrete line $\mathfrak{D}(\mathbf{v}, \mu, \omega)$, if $v_{1}$ and $v_{2}$ are rationnally dependent, then one can suppose ${ }^{14}$ that $\mathbf{v} \in \mathbb{Z}^{2}, \mu \in \mathbb{Z}$ and $\omega \in \mathbb{N}$. Consequently, $\mathfrak{D}(\mathbf{v}, \mu, w)$ is the set of solutions of at most $\omega$ diophantine equations. The arithmetical thickness $w$ is also strongly related to the connectedness of arithmetical discrete lines (see Figures 1(b) and 1(c)).

Theorem 1 ( $k$-minimality and arithmetical discrete lines $\left.{ }^{14}\right)$ The arithmetical discrete line $\mathfrak{D}(\mathbf{v}, \mu, \omega)$ is 0-minimal (resp. 1-minimal) if and only if $\omega=\|\mathbf{v}\|_{1}$ (resp. $\left.\omega=\|\mathbf{v}\|_{\infty}\right)$.

\footnotetext{
* “... le désaccord entre le Discret et le Continu se mesure par un éloignement "infini” pris en compte dans l'un des axiomes fondateurs de la Mathématique. Le concept de nombre entier infiniment grand nous permet d'envisager une Mathématique Discrète Idéale intermédiaire entre la classique Mathématique du Continu et la Mathématique Discrète ; comme la pile d'un pont entre deux rives..."14
} 


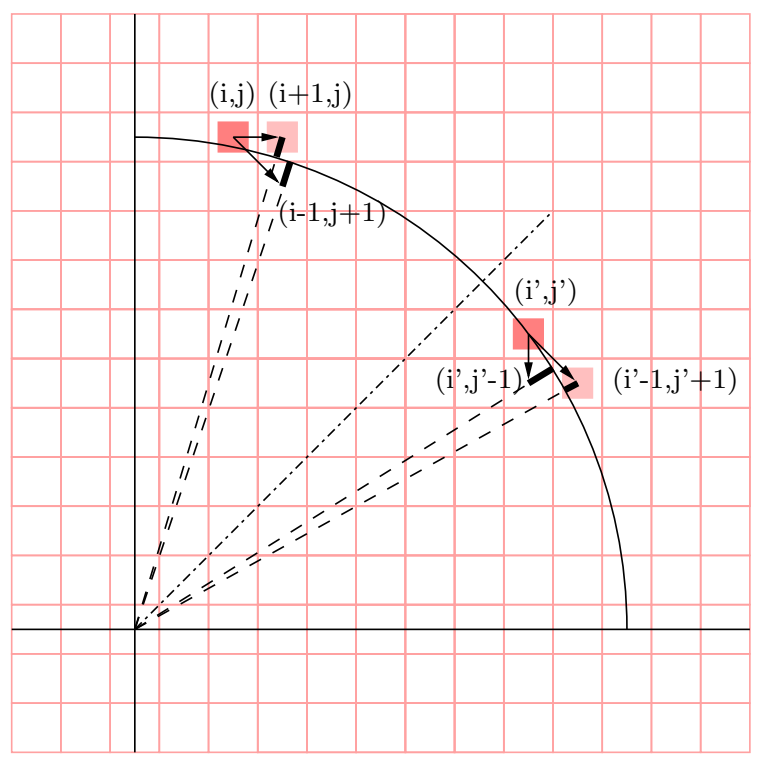

(a) Incremental process of building

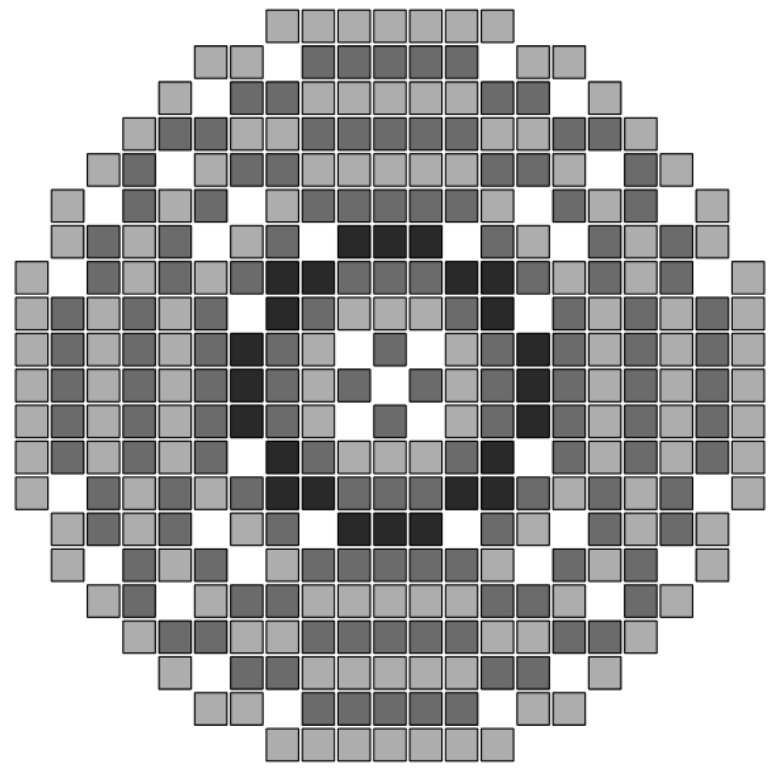

(b) Bresenham's circles of integer radii between 1 and 10 .

Figure 2. Bresensenham's circles.

Definition 4 (Naive ${ }^{14}$ and standard ${ }^{6}$ discrete lines) An arithmetical discrete line $\mathfrak{D}(\mathbf{v}, \mu, \omega)$ is said to be naive (resp. standard) if $\omega=\|\mathbf{v}\|_{\infty}$ (resp. $\left.\omega=\|\mathbf{v}\|_{1}\right)$.

In other words, an arithmetical discrete line $\mathfrak{D}(\mathbf{v}, \mu, \omega)$ is naive (resp. standard) if and only if it is 1-minimal (resp. 0-minimal).

\section{ALREADY KNOWN DISCRETE CIRCLES}

In the present section, we go back over two different visions of discrete circles, an algorithmical one due to J. Bresenham ${ }^{4}$ and an analytical one due to É. Andres. ${ }^{1}$

We first have a look at the algorithmical approach, the oldest and more studied one, before taking an interest in the analytical discrete circles, a vision arising from the arithmetical discrete geometry.

\subsection{Algorithmical Approach}

Required by digital plotters, many circle drawing algorithms, ${ }^{4,10,11,13}$ have been developped. All of them intend to find a thinnest discrete circle, ideally a 1-minimal one, and incrementally return the computed pixels. Moreover, most of them only compute in the first quadrant, the one where the pixels have all positive coordinates. The entire circle is deduced by axial symmetries.

Let us now remind J. Bresenham's algorithm ${ }^{4}$ for drawing discrete circles with centers in $\mathbb{Z}^{2}$ and integer radii. Our choice is motivated by the fact that this discrete circle drawing algorithm is certainly the best known one. In addition, the outputs of the other ones ${ }^{10,11,13}$ are close together.

In the first quadrant, a circle is a decreasing function of the abscissa. Consequently, in order to cover this quadrant clockwise, J. Bresenham's algorithm involves only three unitary moves, namely down, down-right and right (see Figure 2(a)). 
Let $R \in \mathbb{N}$ and let us draw the Bresenham's circle $\mathbb{B}(\mathbf{0}, R)$ of center $\mathbf{O}=(0,0)$ and radius $R$, namely the digitization of the euclidean circle $\mathcal{C}(\mathbf{O}, R)$ of center $\mathbf{O}=(0,0)$ and radius $R$. Let $\Delta$ be the following map:

$$
\begin{aligned}
\Delta: \mathbb{N}^{2} & \longrightarrow \mathbb{Z} \\
(i, j) & \longmapsto(i+1)^{2}+(j-1)^{2}-R^{2} .
\end{aligned}
$$

Then, for each $(i, j) \in \mathbb{N}^{2}, \Delta(i, j) \geq 0$ if and only if the pixel $(i+1, j-1)$ is outside $\mathcal{C}(\mathbf{O}, R)$.

The initial selected pixel is $(0, R)$. Since $R \in \mathbb{N}^{\star}$, one deduces that the pixel $(0, R)$ belongs to $\mathcal{C}(\mathbf{O}, R)$.

Let $(i, j)$ be a computed pixel in the first quadrant. J. Bresenham shows that $\Delta(i, j) \geq 0$ if and only if $(i, j)$ is in the second octant of $\mathbb{Z}^{2}$, that is, the set $\left\{(i, j) \in \mathbb{Z}^{2} \mid 0 \leq i \leq j\right\}$ and it remains two candidates for being the next pixel. Let us select the closest one to $\mathcal{C}(\mathbf{O}, R)$. In the first octant, it is determinated by the difference $\delta_{1}(i, j)$ between the distances from $(i+1, j)$ and $(i+1, j-1)$ to $\mathcal{C}(\mathbf{O}, R)$ :

$$
\delta_{1}(i, j)=\left|(i+1)^{2}+j^{2}-R^{2}\right|-\left|(i+1)^{2}+(j-1)^{2}-R^{2}\right| .
$$

If $\delta_{1}(i, j) \leq 0$ then the pixel $(i+1, j)$ is selected, and so are the pixels $(-i-1, j),(-i-1,-j)$, and $(i+1,-j)$ by symmetries. Otherwise, the pixels $(i+1, j-1),(-i-1, j-1),(-i-1,-j+1),(i+1,-j+1)$ are selected. In the second octant, the same holds for the pixels $(i+1, j-1),(i, j-1)$ and their symmetrical images.

As shown in the present section, J. Bresenham's algorithm allows to draw discrete circles of centers in $\mathbb{Z}^{2}$ and integer radii (see Figure 2(b)). Most of the time, it returns a 1-minimal discrete circle (see Definition 2). Otherwise, the returned discrete circle contains exactly four 1-simple points located on the diagonals (spikes ${ }^{9}$ or sharp corners $^{10}$ ). Z. Kulpa ${ }^{9}$ characterized the concerned radii $R$ :

$$
R^{2}=2\left\lceil\frac{R}{\sqrt{2}}\right\rceil^{2}-\left\lceil\frac{R}{\sqrt{2}}\right\rceil+1
$$

whose first solutions are 4, 11, 134, 373, 4552, 12671, 154634, 430441, 5253004, 14622323, ...

\subsection{Discrete Analytical Circles ${ }^{1}$}

The algorithmical approach of discrete circle drawing $4,10,11,13$ let several questions unsolved. For example, they do not compute a set of discrete circles tiling the discrete space $\mathbb{Z}^{2}$ and do not allow to check whether a point belongs to a given discrete circle.

É. Andres fills partially this lack by introducing the discrete analytical circles. ${ }^{1}$ Roughly speaking, similarly to the arithmetical discrete lines (see Definition 3), the discrete analytical circles are defined as rings depending on a parameter $\omega \in \mathbb{R}_{+}$called the arithmetical thickness (see Figure 3 ).

Definition 5 (Discrete analytical circles ${ }^{1}$ ) The discrete analytical circle $\mathbb{C}(\mathbf{M}, R, \omega)$ of center $\mathbf{M}_{\mathbf{0}}=\left(x_{0}, y_{0}\right) \in$ $R^{2}$, of radius $R \in \mathbb{R}_{+}^{\star}$ and of arithmetical thickness $\omega \in \mathbb{R}_{+}^{\star}$, is the following subset of $\mathbb{Z}^{2}$ defined by:

$$
\mathbb{C}\left(\mathbf{M}_{\mathbf{0}}, R, \omega\right)=\left\{(i, j) \in \mathbb{Z}^{2} \mid\left(R-\frac{\omega}{2}\right)^{2} \leq\left(i-x_{0}\right)^{2}+\left(j-y_{0}\right)^{2}<\left(R+\frac{\omega}{2}\right)^{2}\right\} .
$$

One obtains limited results on the relation between the arithmetical thickness and the connectedness of a discrete analytical circle.

Proposition 1 (Discrete analytical circles and connectedness ${ }^{2}$ ) Let $\mathbb{C}\left(\mathbf{M}_{\mathbf{0}}, R, \omega\right)$ be a discrete analytical circle. If $\omega \geq 1$, then $\mathbb{C}\left(\mathbf{M}_{\mathbf{0}}, R, \omega\right)$ is connected. Moreover, for each point $\mathbf{M}_{\mathbf{0}} \in \mathbb{Z}^{2}$ and for each $\left.\left.R \in\right] 0,1\right]$, the set $\left\{\mathbb{C}\left(\mathbf{M}_{\mathbf{0}}, R+k, 1\right), k \in \mathbb{N}\right\}$ tiles the discrete space $\mathbb{Z}^{2}$.

Definition 6 (Regular discrete circles $\left.^{2}\right)$ Let $\mathbb{C}\left(\mathbf{M}_{\mathbf{0}}, R, \omega\right)$ be a discrete analytical circle. If $w=1$ then $\mathbb{C}\left(\mathbf{M}_{\mathbf{0}}, R, \omega\right)$ is said to be regular (see Figure $3(\mathrm{~b})$ ).

One can notice that, contrary to the thickness of an arithmetical discrete line, the one of a discrete analytical circle does not entirely characterize its connectedness. For instance, if $\omega<1$, then we cannot state in the general case whether $\mathbb{C}\left(\mathbf{M}_{\mathbf{0}}, R, \omega\right)$ is connected. Moreover, when $\omega \geq 1$, we cannot conclude whether $\mathbb{C}\left(\mathbf{M}_{\mathbf{0}}, R, \omega\right)$ is 0-separable, 0-minimal or 1-connected. 


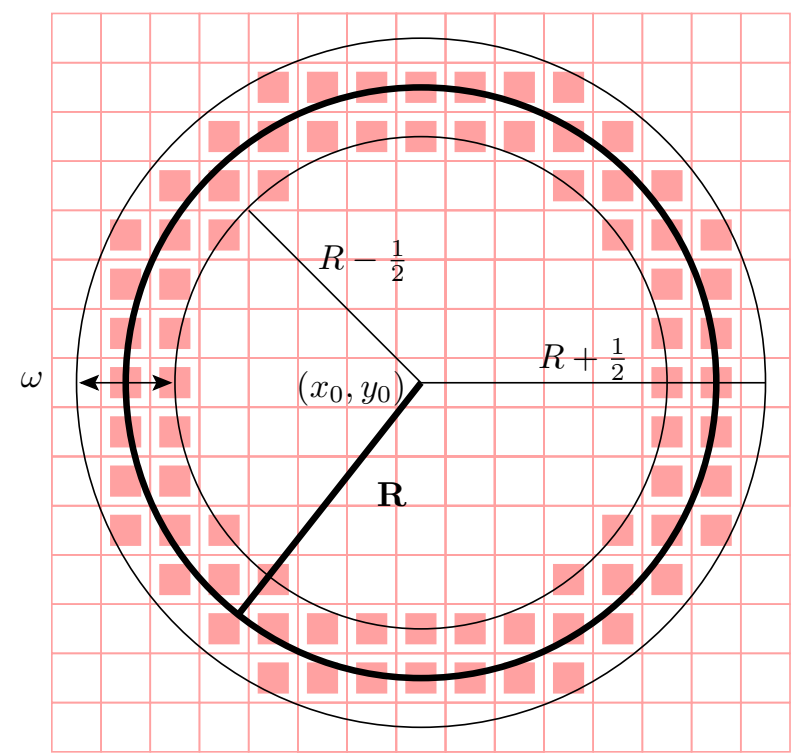

(a) General definition

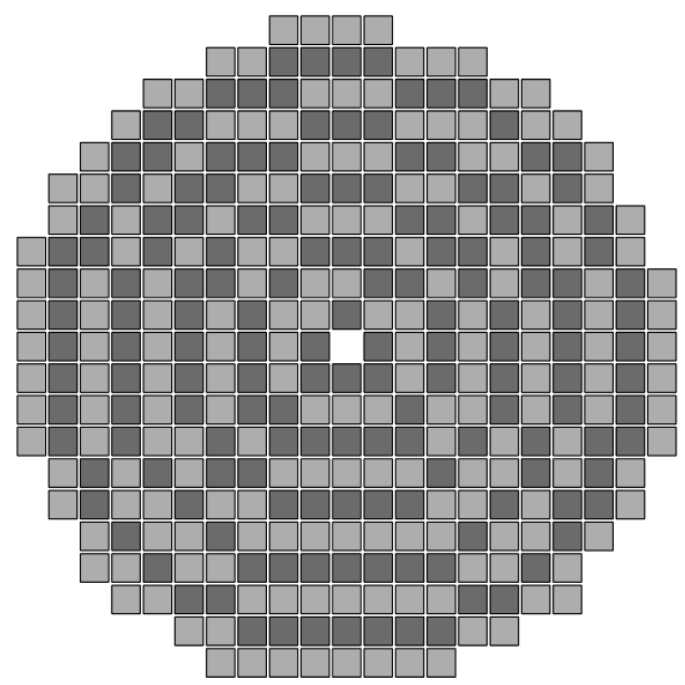

(b) Concentric regular discrete circles of real coordinate center.

Figure 3. Discrete analytical circles.

\section{ARITHMETICAL DISCRETE CURVES: A TANGENTIAL POINT OF VIEW}

In Section 4, we notice that the algorithmical (Section 4.1) and analytical (Section 4.2) approaches do not characterize the same discrete circles. On the one hand, the Bresenham's discrete circles are often 1-minimal but do not tile the discrete space $\mathbb{Z}^{2}$. On the other hand, discrete analytical circles tile $\mathbb{Z}^{2}$ but their thinness is not entirely controled by their parameters. On can see that discrete circles defined by J. Bresenham ${ }^{4}$ and É. Andres ${ }^{1}$ are quite far to satisfy the conditions (i), (ii) and (iii) page 1.

From theorem 1, given an arithmetical discrete line $\mathfrak{D}(\mathbf{v}, \mu, \omega)$ with normal vector $\mathbf{v}=(a, b) \in \mathbb{R}^{2}$, the $k$ connectedness and the $k$-minimality of $\mathfrak{D}(\mathbf{v}, \mu, \omega)$ only depend on its thickness, expressed as a function of $a$ and $b$, namely the coordinates of the vector $\mathbf{v}$. In fact, those coordinates are nothing but the partial derivatives of the linear form $a x+b y+\mu$. In other words, $\mathbf{v}$ is the normal vector of the tangent to the euclidean line with equation $a x+b y+\mu=0$ at each of its points.

Thus, in order to define relevant discrete analogues of non-linear forms, it becomes natural to consider a differential approach. For clarity issues, let us first introduce notation.

Notation. — Let $f: \mathbb{R}^{2} \longrightarrow \mathbb{R}$ be a differentiable function. We denote by $\partial_{x} f: \mathbb{R}^{2} \longrightarrow \mathbb{R}$ and $\partial_{y} f: \mathbb{R}^{2} \longrightarrow$ $\mathbb{R}$ the following functions:

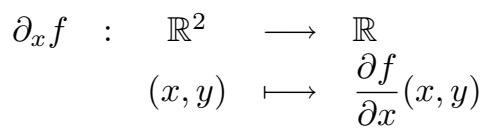

and

$$
\begin{aligned}
& \partial_{y} f: \mathbb{R}^{2} \longrightarrow \mathbb{R} \\
& (x, y) \longmapsto \frac{\partial f}{\partial y}(x, y)
\end{aligned}
$$

Definition 7 (Arithmetical discrete curves) Let $f \in \mathbb{R}[x, y]$ be a polynomial in two indeterminates over $\mathbb{R}$ and let $\omega: \mathbb{R}^{2} \longrightarrow \mathbb{R}$. The arithmetical discrete curve $\mathfrak{C}(f, \omega)$ of equation $f(x, y)=0$ and thickness map $\omega$ is defined by:

$$
\mathfrak{C}(f, \omega)=\left\{(i, j) \in \mathbb{Z}^{2} \mid-\frac{\omega\left(\partial_{x} f(i, j), \partial_{y} f(i, j)\right)}{2} \leq f(i, j)<\frac{\omega\left(\partial_{x} f(i, j), \partial_{y} f(i, j)\right)}{2}\right\} .
$$


Let us notice that the arithmetical discrete line of normal vector $\mathbf{v}=(a, b)$ is canonically an arithmetical discrete curve and, for all $(i, j) \in \mathbb{Z}^{2}$,

$$
\omega\left(\partial_{x} f(i, j), \partial_{y} f(i, j)\right)=\omega(a, b)
$$

and $\omega$ can be replaced by a constant function.

As noticed for arithmetical discrete lines, the choice of inequalities in (6) is arbitrary and one can choose the opposite convention. The arithmetical discrete curve $\widetilde{\mathfrak{C}}(f, \omega)$ of equation $f(x, y)=0$ and thickness map $\omega$ is defined by:

$$
\widetilde{\mathfrak{C}}(f, \omega)=\left\{(i, j) \in \mathbb{Z}^{2} \mid-\frac{\omega\left(\partial_{x} f(i, j), \partial_{y} f(i, j)\right)}{2}<f(i, j) \leq \frac{\omega\left(\partial_{x} f(i, j), \partial_{y} f(i, j)\right)}{2}\right\}
$$

\section{A PARTICULAR CASE: THE ARITHMETICAL DISCRETE CIRCLES}

In the present Section, we focus on a particular case of arithmetical discrete curves. In fact, from the quadratic form $f,\left(x-x_{0}\right)^{2}+\left(y-y_{0}\right)^{2}-R^{2}$ for all $(x, y) \in \mathbb{R}^{2}$ and $\left(x_{0}, y_{0}, R\right) \in \mathbb{R}^{2} \times \mathbb{R}_{+}$, we investigate the arithmetical discrete circles.

\subsection{General Definition}

Definition 8 (Inner and outer discrete circles) Let $\mathbf{M}_{\mathbf{0}}=\left(x_{0}, y_{0}\right)$ be a point of the discret space $\mathbb{Z}^{2}, R \in$ $\mathbb{R}_{+}$and $\omega: \mathbb{R}^{2} \longrightarrow \mathbb{R}$.

The inner discrete circle $\mathfrak{C}\left(\mathbf{M}_{\mathbf{0}}, R, \omega\right)$ of center $\mathbf{M}_{\mathbf{0}}$, radius $R$ and thickness function $\omega$ is the set of point $(i, j)$ with integer coordinates defined by:

$$
\mathfrak{C}\left(\mathbf{M}_{\mathbf{0}}, R, \omega\right)=\left\{(i, j) \in \mathbb{Z}^{2} \mid-\frac{\omega(2 i, 2 j)}{2} \leq f(i, j)<\frac{\omega(2 i, 2 j)}{2}\right\}
$$

The outer discrete circle $\widetilde{\mathfrak{C}}\left(\mathbf{M}_{\mathbf{0}}, R, \omega\right)$ of center $\mathbf{M}_{\mathbf{0}}$, radius $R$ and thickness function $\omega$ is the set of point $(i, j)$ with integer coordinates defined by:

$$
\widetilde{\mathfrak{C}}\left(\mathbf{M}_{\mathbf{0}}, R, \omega\right)=\left\{(i, j) \in \mathbb{Z}^{2} \mid-\frac{\omega(2 i, 2 j)}{2}<f(i, j) \leq \frac{\omega(2 i, 2 j)}{2}\right\} .
$$

We select appropriate thickness function to highlight relevant classes of arithmetical discrete circles. In the one hand, we provide the regular discretes circle ${ }^{1}$ of integer parameters and the Bresenham's circles ${ }^{4}$ with aritmetical definitions. In the other hand, we characterize new discrete circles, the 1-minimal and the 0-minimal ones.

Let us bring up again the relevant classes of arithmetical discrete lines (see Definition 4). The thickness of a naive (resp. standard) discrete line of normal vector $\mathbf{v}$ is the $\|\mathbf{v}\|_{\infty}$ (resp. $\|\mathbf{v}\|_{1}$ ). The norms seems to control the connectedness of the arithmetical discrete lines. Moreover, the definition of the discrete analytical circles imply the same idea with the $\|\cdot\|_{2}$ :

$$
R-\frac{1}{2} \leq\left\|\left(i-x_{0}, j-y_{0}\right)\right\|_{2}<R+\frac{1}{2} \Leftrightarrow\left(R-\frac{1}{2}\right)^{2} \leq\left(i-x_{0}\right)^{2}+\left(j-y_{0}\right)^{2}<\left(R+\frac{1}{2}\right)^{2} .
$$

Notation. - We denote by $\omega_{k}: \mathbb{Z}^{2} \longrightarrow \mathbb{R}_{+}$, the thickness function, related to the norm $\|\cdot\|_{k}$, as following:

$$
\begin{aligned}
\omega_{k}: \mathbb{Z}^{2} & \longrightarrow \mathbb{R}_{+} \\
(i, j) & \longmapsto\left\|\left(\frac{\partial f(i, j)}{\partial i}, \frac{\partial f(i, j)}{\partial j}\right)\right\|_{k} .
\end{aligned}
$$




\subsection{Characterizations of Already Known Discrete Circles}

The thickness function $\omega_{2}$ provides the regular discrete circle $^{1}$ of integer parameters with a new arithmetical definition (see Figure 4(a)).

Proposition 2 (Regular discrete circles) Let $\mathbf{N}_{\mathbf{0}}=\left(i_{0}, j_{0}\right) \in \mathbb{Z}^{2}$ and $R \in \mathbb{N}$. The regular discrete circle ${ }^{1}$ $\mathbb{C}\left(\mathbf{N}_{\mathbf{0}}, R, 1\right)$ is the arithmetical discrete circle $\mathfrak{C}\left(\mathbf{N}_{\mathbf{0}}, R, \omega_{2}\right)$ but also the arithmetical discrete circle $\widetilde{\mathfrak{C}}\left(\mathbf{N}_{\mathbf{0}}, R, \omega_{2}\right)$ :

$$
\mathfrak{C}\left(\mathbf{N}_{\mathbf{0}}, R, \omega_{2}\right)=\mathbb{C}\left(\mathbf{N}_{\mathbf{0}}, R, 1\right)=\widetilde{\mathfrak{C}}\left(\mathbf{N}_{\mathbf{0}}, R, \omega_{2}\right)
$$

Proof. On the one hand, the regular discrete $\operatorname{circle}^{1} \mathbb{C}\left(\mathbf{N}_{\mathbf{0}}, R, 1\right)$ is characterized as follow:

$$
\mathbb{C}\left(\mathbf{N}_{\mathbf{0}}, R, \omega\right)=\left\{(i, j) \in \mathbb{Z}^{2} \mid\left(R-\frac{1}{2}\right)^{2} \leq\left(i-i_{0}\right)^{2}+\left(j-i_{0}\right)^{2}<\left(R+\frac{1}{2}\right)^{2}\right\} .
$$

Since $\mathbf{N}_{\mathbf{0}}=\left(i_{0}, j_{0}\right) \in \mathbb{Z}^{2}$ and $R \in \mathbb{N}$, the double inequality defining the set above amounts to: $-R<\left(i-i_{0}\right)^{2}+$ $\left(j-i_{0}\right)^{2}-R^{2}<R+1$.

On the other hand, the arithmetical discrete circle $\mathfrak{C}\left(\mathbf{N}_{\mathbf{0}}, R, \omega_{2}\right)$ is characterized as follow:

$$
\mathfrak{C}\left(\mathbf{N}_{\mathbf{0}}, R, \omega\right)=\left\{(i, j) \in \mathbb{Z}^{2} \mid-\sqrt{\left(i-i_{0}\right)^{2}+\left(j-j_{0}\right)^{2}} \leq\left(i-i_{0}\right)^{2}+\left(j-j_{0}\right)^{2}-R^{2}<\sqrt{\left(i-i_{0}\right)^{2}+\left(j-j_{0}\right)^{2}}\right\} .
$$

Let $k=\sqrt{\left(i-i_{0}\right)^{2}+\left(j-j_{0}\right)^{2}}$ and $g_{1}: \mathbb{N} \longrightarrow \mathbb{R}$ be the map:

$$
\begin{aligned}
g_{1}: \mathbb{R} & \longrightarrow \mathbb{R} \\
k & \longmapsto k^{2}+k-R .
\end{aligned}
$$

$g_{1}$ is an increasing function. Moreover, $g_{1}(R-1)<0$ and $g_{1}(R)>0$. Let $k_{\text {min }} \in R$ such that $g_{1}\left(k_{\text {min }}\right)=0$. We have $g_{1}(R-1)<g_{1}\left(k_{\text {min }}\right)<g_{1}(R)$ and then, $R<k_{\min }<R-1$. Since $\left(i-i_{0}\right)^{2}+\left(j-i_{0}\right)^{2}-R^{2}$ is an integer, we look for integer bounds. Such an assumption leads to the lower bound $-R<\left(i-i_{0}\right)^{2}+\left(j-j_{0}\right)^{2}-R^{2}$. Similarly, the upper bound $\left(i-i_{0}\right)^{2}+\left(j-j_{0}\right)^{2}-R^{2}<R+1$ is obtained and finally, $\mathbb{C}\left(\mathbf{N}_{\mathbf{0}}, R, 1\right)=\mathfrak{C}\left(\mathbf{N}_{\mathbf{0}}, R, \omega_{2}\right)$.

We can in a similar way prove $\mathbb{C}\left(\mathbf{N}_{\mathbf{0}}, R, 1\right)=\widetilde{\mathfrak{C}}\left(\mathbf{N}_{\mathbf{0}}, R, \omega_{2}\right)$.

The thickness function $\omega_{\infty}$ provides the Bresenham's circles with a new characterization. As far as we know, this is the first arithmetical definition of them. The original definition of the Bresenham's circle compute only circles of integer radius. Here, we use its extension to integral square radius introduced by M. McIlroy. ${ }^{10}$

Proposition 3 (Arithmetical characterization of the Bresenham's circle) Let $\mathbf{N}_{\mathbf{0}}=\left(i_{0}, j_{0}\right) \in \mathbb{Z}^{2}$ and $R$ such that $R^{2} \in \mathbb{N}$. The arithmetical discrete circle $\mathfrak{C}\left(\mathbf{N}_{\mathbf{0}}, R, \omega_{\infty}\right)$ is the Bresenham's circle $\mathbb{B}\left(\mathbf{N}_{\mathbf{0}}, R\right)$ :

$$
\mathfrak{C}\left(\mathbf{N}_{\mathbf{0}}, R, \omega_{\infty}\right)=\mathbb{B}\left(\mathbf{N}_{\mathbf{0}}, R\right)
$$

Proof. First of all, The arithmetical discrete circle $\mathfrak{C}\left(\mathbf{N}_{\mathbf{0}}, R, \omega_{\infty}\right)$ contains the pixel initially selected by the bresenham's algorithm, $(0, R)$. Now we show that the both definitions respect the same criterion to determine which will be the next pixel of the discrete circle. Let $\mathbf{N}=(i, j) \in \mathbb{N}^{2}$ be a pixel of the arithmetical discrete circle $\mathfrak{C}\left(\mathbf{N}_{\mathbf{0}}, R, \omega_{\infty}\right)$ such that $0 \leq j \leq i$, namely in the second octant. Let consider that we cover the second octant clockwise. Because of our definition, and with $I=i-i_{0}, J=j-j_{0}$, two pixels are reachable:

- the pixel $(i+1, j-1)$ belongs to $\mathfrak{C}\left(N_{0}, R, \omega_{\infty}\right)$ if and only if $-2 I+J-1 \leq I^{2}+J^{2}-R^{2}$,

- the pixel $(i+1, j)$ belongs to $\mathfrak{C}\left(N_{0}, R, \omega_{\infty}\right)$ if and only if $I^{2}+J^{2}-R^{2}<-2 I+J-1$. 


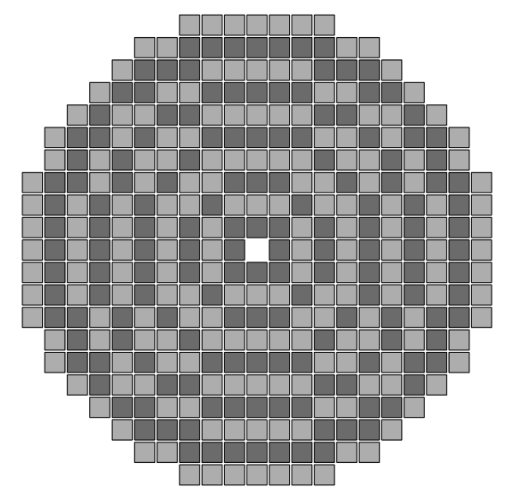

(a) Regular discrete circles of integer radii between 1 et 10 .

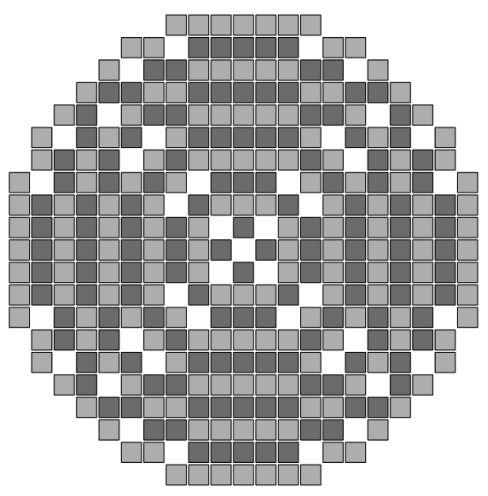

(b) Naive discrete circles of integer radii between 1 and 10 .

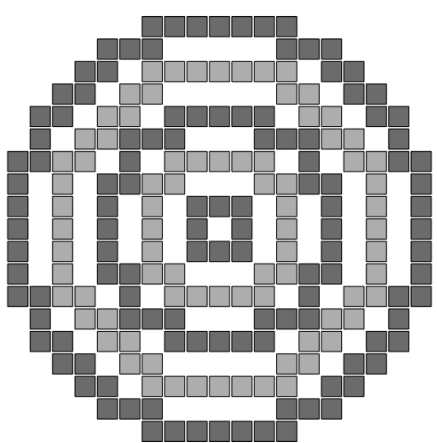

(c) Standard discrete circles of radii $1,3,5,7$ and 9 .

Figure 4. Some classes of discrete circles we obtain.

Thus, the sign of the term $I^{2}+J^{2}-R^{2}+2 I-J+1$ determined the next pixel to be selected.

Bresenham's algorithm uses as selection criterion the minimization of the distance between the euclidean circle and the next pixel. It compares the two distances:

$$
\begin{aligned}
& \delta_{2}(i, j)=\left[(I+1)^{2}+(J)^{2}-R^{2}\right]+\left[(I+1)^{2}+(J-1)^{2}-R^{2}\right] \\
& \frac{\delta_{2}(i, j)}{2}=I^{2}+J^{2}-R^{2}+2 I-J+\frac{3}{2}
\end{aligned}
$$

$I^{2}+J^{2}-R^{2}+2 I-J$ is an integer, thus the sign of the term $I^{2}+J^{2}-R^{2}+2 I-J+1$ is equivalent to the one of $I^{2}+J^{2}-R^{2}+2 I-J+\frac{3}{2}$ and determines the next pixel to be selected.

Similarly, in the first octant, the selection criterion is the same. Then, starting from the same initialisation and computing with the same criterion, $\mathfrak{C}\left(\mathbf{N}_{\mathbf{0}}, R, \omega_{\infty}\right)=\mathbb{B}\left(\mathbf{N}_{\mathbf{0}}, R\right)$.

\subsection{New Classes of Discrete Circles}

Now, we introduce two new definitions of discrete circles related to their $k$-minimality.

Definition 9 (Naive discrete circles) By analogy with discrete lines, 1-minimal discrete circles are said to be naive.

Definition 10 (Standard discrete circles) By analogy with discret lines, 0-minimal discrete circles are said to be standard.

The thickness function $\omega_{\infty}$ allows to define naive discrete circles (see Figure 4(b)).

Proposition 4 (Naive discrete circles) Let $\mathbf{N}_{\mathbf{0}}=\left(i_{0}, j_{0}\right) \in \mathbb{Z}^{2}, R \in \mathbb{N}$ and $\Delta=R^{2}-2\left\lceil\frac{R}{\sqrt{2}}\right\rceil^{2}+\left\lceil\frac{R}{\sqrt{2}}\right\rceil$.

1. The inner arithmetical discrete circle $\mathfrak{C}\left(\mathbf{N}_{\mathbf{0}}, R, \omega_{\infty}\right)$ is naive if and only if $\Delta \neq 0$. 
2. The outer arithmetical discrete circle $\widetilde{\mathfrak{C}}\left(\mathbf{N}_{\mathbf{0}}, R, \omega_{\infty}\right)$ is naive if and only if $\Delta \neq 1$.

So, at least one of them is a naive discrete circle.

Proof. First, we investigate the inner arithmetical discrete circle $\mathfrak{C}\left(\mathbf{N}_{\mathbf{0}}, R, \omega_{\infty}\right)$. Since it is the Bresenham's circle $\mathbb{B}\left(\mathbf{N}_{\mathbf{0}}, R\right)$, it is 0 -minimal except for radii verifying the equality 4 . Then, the equivalence 1 is true.

Second, we investigate the outer arithmetical discrete circle $\widetilde{\mathfrak{C}}\left(\mathbf{N}_{\mathbf{0}}, R, \omega_{\infty}\right)$. we have $\widetilde{\mathfrak{C}}\left(\mathbf{N}_{\mathbf{0}}, R, \omega_{\infty}\right)=\mathfrak{C}\left(\mathbf{N}_{\mathbf{0}}, \sqrt{R^{2}+1}, \omega_{\infty}\right)$. Hence, $\widetilde{\mathfrak{C}}\left(\mathbf{N}_{\mathbf{0}}, R, \omega_{\infty}\right)$ is the Bresenham's circle $\mathbb{B}\left(\mathbf{N}_{\mathbf{0}}, \sqrt{R^{2}+1}\right)$ of center $\mathbf{N}_{\mathbf{0}}$ and radius $\sqrt{R^{2}+1}\left({\sqrt{R^{2}+1}}^{2}\right.$ is an integer). One shows this discrete circle is 1-minimal except for radii verifying: $R^{2}=2\left\lceil\frac{R}{\sqrt{2}}\right\rceil^{2}-\left\lceil\frac{R}{\sqrt{2}}\right\rceil$. Thus, the equivalence 2 is true.

Similarly, the thickness function $\omega_{1}$ allows to define standard discrete circles (see Figure 4(c)).

Proposition 5 (Standard discrete circles) Let $\mathbf{M}_{\mathbf{0}}=\left(x_{0}, y_{0}\right) \in \mathbb{R}^{2}$ and $R \in \mathbb{R}_{+}$. The arithmetical discrete circles $\mathfrak{C}\left(\mathbf{M}_{\mathbf{0}}, R, \omega_{1}\right)$ and $\widetilde{\mathfrak{C}}\left(\mathbf{M}_{\mathbf{0}}, R, \omega_{1}\right)$ are standard discrete circles.

Proof. The arithmetical discrete circle $\mathfrak{C}\left(\mathbf{M}_{\mathbf{0}}, R, \omega_{1}\right)$ includes the arithmetical discrete circles $\mathfrak{C}\left(\mathbf{M}_{\mathbf{0}}, R, \omega_{\infty}\right)$, since for all pixel $\mathbf{N}=(i, j), \omega_{1}(2 i, 2 j)>\omega_{\infty}(2 i, 2 j)$, and is at least 1-minimal. With no loss of generality, let consider we cover clockwise the first quadrant of the inner arithmetical discrete circle $\mathfrak{C}\left(\mathbf{M}_{\mathbf{0}}, R, \omega_{1}\right)$. A pixel $N=(i, j) \in \mathfrak{C}^{-}\left(M_{0}, R, \omega_{1}\right)$ satisfies the following double diophantine inequality:

$$
-X-Y \leq X^{2}+Y^{2}-R^{2}<X+Y,
$$

where $X=i-x_{0}$ and $Y=j-y_{0}$. Under our assumptions, only three pixels, $(i+1, j),(i+1, j-1)$ and $(i, j-1)$, are canditates for being the next clockwise one belonging to the discrete circle $\mathfrak{C}\left(\mathbf{M}_{\mathbf{0}}, R, \omega_{1}\right)$. It is sufficient that the pixel $(i+1, j)$ or the pixel $(i, j-1)$ belongs to the discrete circle to have 0-separability (1-connectedness) and it is necessary that the both do not belong simultaneously to the discrete circle to avoid 0-simple points. From equation 6.3 , we obtain the membership for respectively the pixels $(i+1, j)$ and $(i, j-1)$ of the circle:

$$
-X-3 Y+1 \leq X^{2}+(Y-1)^{2}-R^{2}<\begin{aligned}
& X-Y+1 \leq(X+1)^{2}+Y^{2}-R^{2}<3 X+Y+1 \\
& X-Y+1
\end{aligned}
$$

The two inequalities could not be simultaneously valid, but one of the both is always verified. So, standard discrete circles are 0-minimal.

Same study leads to same results for outer standard discrete circles. $\quad$

\section{CONCLUSION}

In the present paper, we introduce a general arithmetical characterization of discrete curves including and extending the definition of the arithmetical discrete lines. ${ }^{14}$

The key point of our approach is the thickness function. As far as we now, this parameter has always been considered as a constant. It leads to a wide knowledge of discrete line ${ }^{14}$ and partially improves the knowledge of discrete circles. $^{1}$ In the present paper, we decide to consider the thickness as a function of the local derivatives of the curve. It doesn't throw results on discrete lines back into question and improves again the characterization of discrete circles.

In fact, in the particular case of discrete circles with integer parameters, our definition includes the Bresenham's circles ${ }^{4}$ and the analytical discrete circles. ${ }^{1}$ Moreover, we reach new classes of discrete circles, the standard and naive ones. Similarly to arithmetical discrete lines, ${ }^{14}$ all these characterizations are obtained by selecting the usual norms as thickness functions. We achieve the aim we state at the beginning of the present paper, namely define discrete circles sharing properties i, ii and iii (see page 1) with the euclidean circle.

In the more general case of discrete circles with real parameters, our definition only characterize standard discrete circles. But the definition of regular discrete circles by E. Andres ${ }^{1}$ is valid for any center with real 


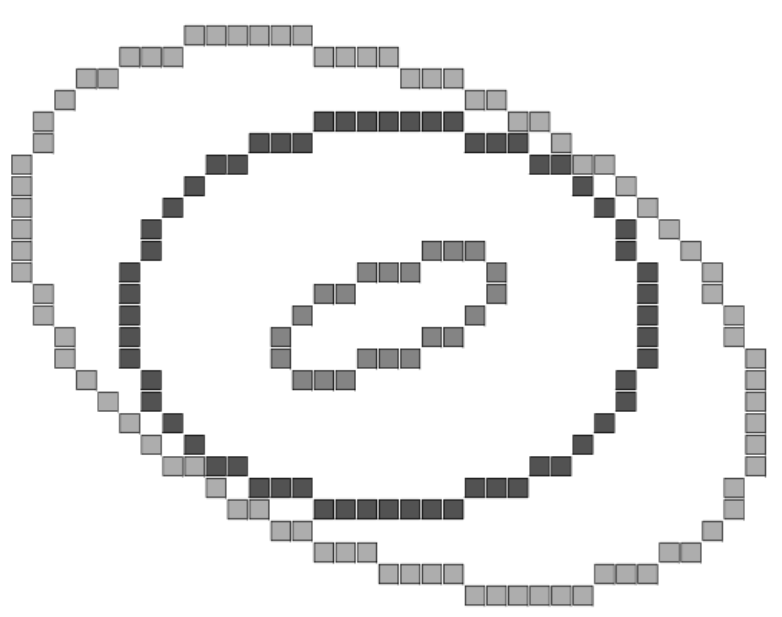

(a) Naive discrete ellipses.

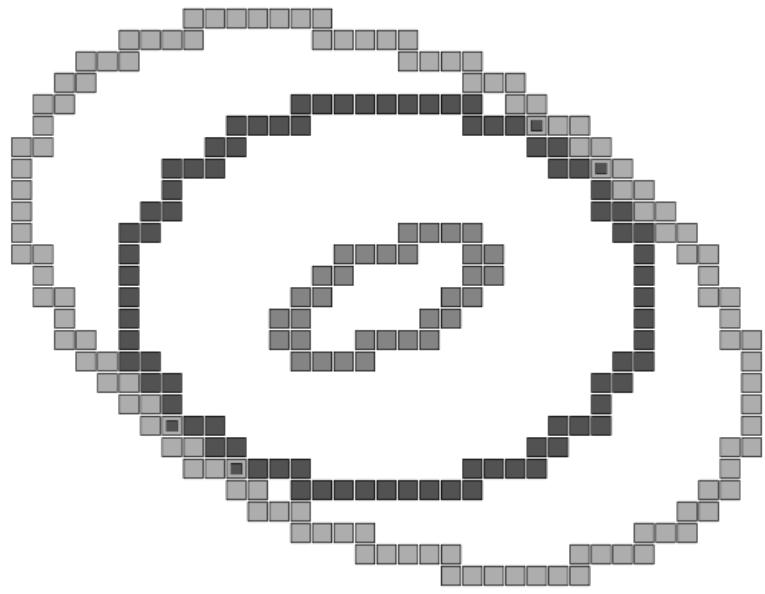

(b) Standard discrete ellipses.

Figure 5. Discrete Ellipses with integer parameters.

coordinates and any real radius, just as Bresenham's circles ${ }^{4}$ since S. Pham ${ }^{12}$ extended them. Tangents can be not an enough accurate approximation of circles. In our next papers we will try to overcome such a limit.

Finally, our approach is valid for polynomial curves and does not dependent on the two-dimensional space. It is thus natural to investigate these both directions. regular discrete representations of most of the curves do not make any sense.then, Figure 5 shows only naive and standard discrete ellipses derivated from our definition. In Figure 5(a), naive discrete ellipses are drawn. In the more general case of polynomial curves, our definition does no characterize naive discrete representation since the two candidates $\mathbb{C}$ and $\widetilde{\mathbb{C}}$, can both have 1-simple points. In figure 5(b), one of the drawing contains 0-simple points and is no more a standard discrete representation as expected. This problem comes from the poor resolution of the discrete lattice compared to the local curvature of the euclidean ellipse. Figure 6 presents some results on spheres. It is possible to recover the regular discrete spheres $^{2}$ of E. Andres, figure 6(b) or, to define the thinnest discrete spheres, mamely the 1-minimal ones, figure $6(a)$. In the same way, the different hyperspheres, naive, regular and standard, are reachable.

\section{REFERENCES}

1. Andres, E. Discrete circles, rings and spheres. Computers \& Graphics 18, 5 (1994), 695-706.

2. Andres, E., AND JACOB, M.-A. The discrete analytical hyperspheres. IEEE Transactions on Visualization and Computer Graphics 3, 1 (1997), 75-86.

3. Bresenham, J. Algorithm for computer control of a digital plotter. IBM Systems Journal 4, 1 (1965), $25-30$.

4. Bresenham, J. A linear algorithm for incremental digital display of circular arcs. Commununication of the ACM 20, 2 (February 1977), 100-106.

5. Debled-Rennesson, I., And Reveillès, J.-P. A linear algorithm for segmentation of digital curves. International Journal on Pattern Recognition and Artificial Intelligence (IJPRAI) 9, 4 (1995), 635-662.

6. FranÇOn, J. Arithmetic planes and combinatorial manifolds. In Actes du 5ème colloque DGCI (september 1995).

7. Freeman, H. Computer processing of line-drawing images. ACM Computing Surveys 6, 1 (1974), 57-97.

8. Hung, S. H. Y. On the straightness of digital arcs. In IEEE Transactions on Pattern Analysis and Machine Intelligence (1985), vol. PAMI-7, pp. 203-215. 


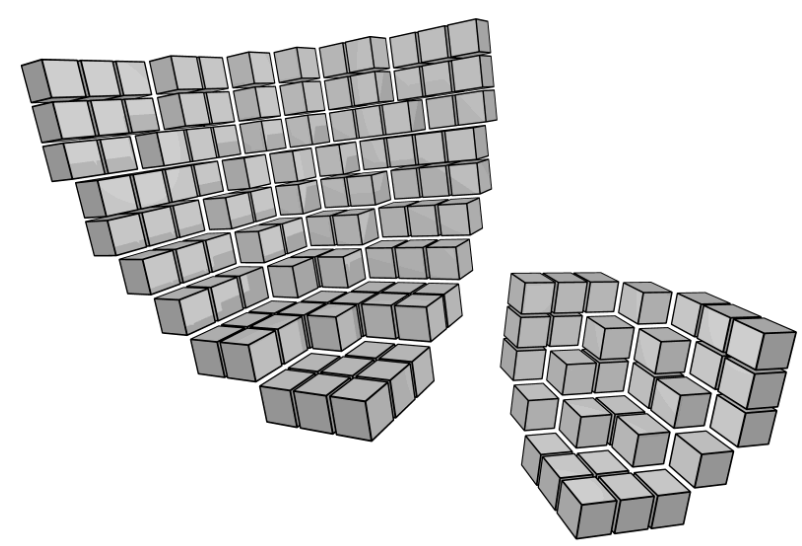

(a) Eighth of thinnest discrete spheres.

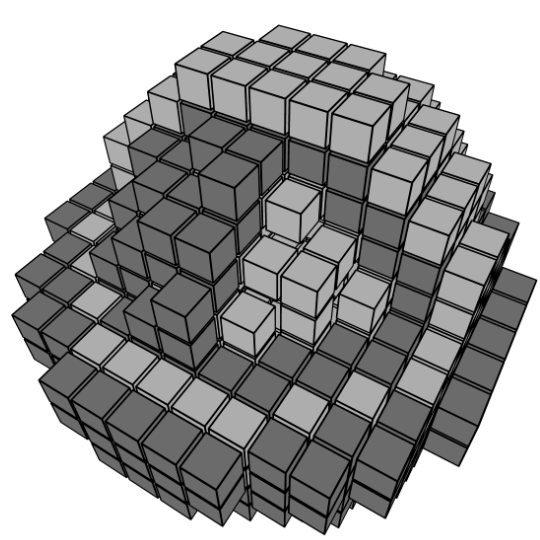

(b) Regular discrete spheres tiling the space.

Figure 6. Spheres with integer parameters.

9. Kulpa, Z. On the properties of discrete circles, rings, and disks. Computer Graphics and Image Processing 10 (1979), 348-365.

10. McIlroy, M. Best approximate circles on integer grids. ACM Transactions on Graphics 2, 4 (October 1983), 237-263.

11. Paterson, T. Circles and the digital differential analyzer. Dr Dobb's Journal : Software Tools 15, 7 (1990), 30, 32, 34-35, 96.

12. Pham, S. Digital circles with non-lattice point centers. The Visual Computer 9, 1 (1992), 1-24.

13. Pitteway, M. Integer circles, etc. - some further thoughts. Computer Graphics and Image Processing 3 (1974), 262-265.

14. Reveillès, J.-P. Géométrie discrète, calcul en nombres entiers et algorithmique. Thèse d'Etat, Université Louis Pasteur, Strasbourg, 1991.

15. Rosenfeld, A. Digital straight lines segments. In IEEE Transactions on Computers (1974), pp. 1264-1369. 